\title{
Does Gross Margin Examination Reduce Re-excision Rate in Breast Conservation for Invasive Carcinoma? CALLER Review
}

\author{
Suzanne Hoekstra ${ }^{1}$ (D), Diane Stoller ${ }^{1}$ (D), Haya Raef ${ }^{2}$ (]) \\ ${ }^{1}$ Mercy Hospital, Breast Care Specialists of Maine, Portland, ME, USA \\ ${ }^{2}$ Tufts University, Boston, MA, USA
}

\begin{abstract}
Objective: Determine if Gross Margin Examination reduces margin re-excision rate. Our institutional practice is to perform Gross Margin Examination (GME) with Real-time re-excision (RRE) for all breast conservation specimens with Invasive Carcinoma.

Materials and Methods: Chart review was done to determine if this practice is helpful. 51 CALLER charts were reviewed from December 2016 to December 2017.

Results: Thirty-three underwent margin RRE based on the GME. 11 had cancer in the re-resected margin, 6 of which were cleared with the RRE. The other 5 were reoperated on to clear the margin because on final pathology a margin other than the re-resected margin was positive for malignancy. GME was helpful in preventing reoperation in $55 \%$. None of the remaining 22 patients receiving were found to have a positive margin on final pathology, with 1.6 margins on average re-resected. 13/18 patients did not have RRE and had a final clear margin, but of the other 5, final margin was positive for DCIS in 2 and Invasive Cancer in 3. GME missed invasive disease at the margin in 3 of these 18 patients.

Conclusion: GME was helpful in preventing reoperation in 6 of 11 patients who would have had a positive margin. However, this resulted in the unnecessary removal of additional normal breast tissue in 22 patients. 3 patients' positive margins were missed with GME and required reoperation. 13 patients were able to avoid re-excision and 11 were able to clear their margin in real-time, improving outcomes 24/51 patients. GME therefore does appear useful.
\end{abstract}

Keywords: CALLER, gross margin examination, re-excision

Cite this articles as: Hoekstra S, Stoller D, Raef H. Does Gross Margin Examination Reduce Re-excision Rate in Breast Conservation for Invasive Carcinoma? CALLER Review. Eur J Breast Health 2020; 16(3): 198-200.

\section{Introduction}

Breast-conserving surgery (BCS) is the established standard approach for women with early-stage invasive breast cancers. During BCS, the breast tumor is excised with negative margins, yielding a better cosmetic result. Various factors are associated with tumor recurrence after BCS, including margin status, which has been shown to be a very important prognostic factor for local recurrence (1). A positive margin is correlated with a twofold increase in ipsilateral breast tumor recurrence (2). This risk is not completely eliminated with the addition of local or systemic adjuvant therapy. In addition, patients who develop local recurrence have an increased risk of distant disease, which can impact survival (3).

Delays in adjuvant therapy can also occur when additional surgical procedures are needed to obtain clear margins after breast conservation. Cosmesis is also impacted, and up to $50 \%$ of patients requiring multiple re-excisions will opt for completion mastectomy (4, 5).

The assessment of lumpectomy margins is an ongoing issue for breast surgeons. Various techniques and technology have been utilized in an attempt to reduce margin re-excision rates. These include imaging techniques such as margin scans, and specimen radiograph and pathological assessment such as frozen section or cytology. To date, none of these intraoperative approaches have been successful in reliably identifying clear margins, resulting in a global re-excision rate of approximately $25 \%$ (6). Intraoperative pathologic techniques have the highest sensitivity and specificity on meta-analysis (7). 
The simplest intraoperative pathological technique for evaluating lumpectomy margins is examination of the specimen for gross evidence of the tumor. This method is quick, inexpensive, simple, and any additional suspicious margins can be immediately resected.

We sought to determine if our institution's protocol for intraoperative gross margin assessment was successful in reducing the number of patients requiring re-operation.

\section{Materials and Methods}

In 2015, the American Society of Breast Surgeons held a multidisciplinary consensus conference entitled a "Collaborative Attempt to Lower Lumpectomy Reoperation rates" (CALLER) and composed a "toolbox" of options to reduce lumpectomy reoperations (8). They then offered surgeons the opportunity to participate in the CALLER Registry, a one-year period during which patients undergoing breast conservation were entered into a special section of the Mastery of Breast Surgery where certain data points regarding re-excision were captured. We performed a retrospective analysis of our institution's patients entered in the CALLER registry during this time.

The most recent consensus for a clear margin for invasive cancer is "no tumor on ink," and this was utilized as our definition of a clear margin for patients with invasive disease (9). We reviewed the operative notes and surgical pathology for all CALLER registry patients. Notation was made regarding the results of intraoperative margin assessment as well as whether reoperation was needed, and why.

\section{Table 1. Patient cohort}

$\begin{array}{lc}\text { Number of patients } & 51 \\ \text { Median age group } & 60-69 \\ \text { Median tumor size group (mm) } & 10-19 \mathrm{~mm} \\ \text { ER positivity } & 43(84 \%) \\ \text { Triple negative } & 5(10 \%)\end{array}$

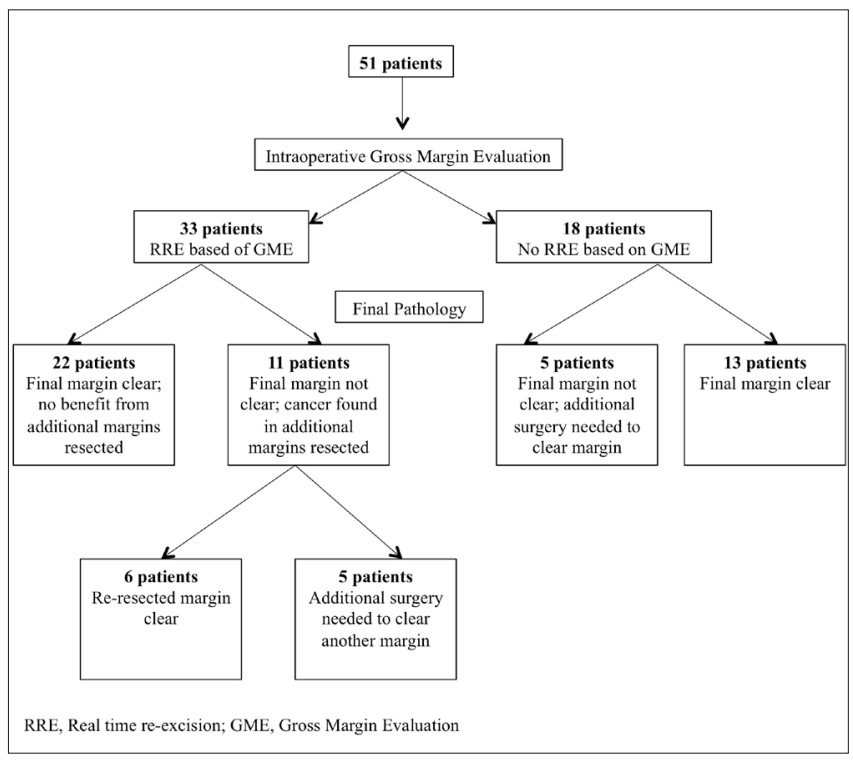

Figure 1. Summary of patient outcomes following gross margin evaluation
Our institutional standard of care is to perform gross margin evaluation (GME) of all lumpectomy specimens done for invasive breast cancer. Each lumpectomy specimen is removed, marked with sutures for orientation, and sent fresh to the pathologist for examination. All lumpectomy specimens were evaluated by a single pathologist. The pathologist inks and sections the specimen. The sections are grossly examined to determine if the tumor appears to be close to or abutting a margin. The pathologist then calls into the operating room and reports the findings, making a recommendation about any margins that might grossly appear to benefit from re-excision. No tissue is frozen or evaluated microscopically. Based on this evaluation, additional margins may be re-resected in real time (RRE) in hopes of avoiding a second operation.

\section{Results}

A total of 61 patients were entered into the CALLER Registry from December 2016 to December 2017. 10 of these patients were excluded because they had pure DCIS, resulting in a total of 51 cases of invasive disease analyzed for review. Information on the patient cohort is provided in Table 1 .

Of these 51 patients, 33 (65\%) underwent margin RRE based on the results of the GME. 11 were found to have cancer in the newly resected margin, and 6 of these had the margin cleared with the RRE. However, the other 5 required a second surgical procedure to completely clear the margins. In all 5 cases this was due to a positive margin other than the one re-excised based on GME based on final pathology. Thus, the GME was helpful in preventing a second operation in only 6 of the 11 patients who had a real-time re-excision.

However, of the other 22 patients who were recommended for and received RRE based on GME, none were found to have a positive margin on the final pathology. The average number of margins resected on these patients was 1.6 as the majority had 1 or 2 margins resected. Thus, the GME did not actually improve the outcome for these patients and increased tissue removed and operative time.

Therefore, of the 33 patients who did receive RRE based on GME, only $6(18 \%)$ of these received a true benefit from the protocol.

Conversely, 18 patients were not recommended to undergo RRE based on GME. Of these patients, the margin was clear on final pathology report in $13(72 \%)$. However, the remaining 5 patients were found to have positive margins at final pathology. Two of these were for DCIS and 3 were for invasive cancer. All but one underwent additional surgery to clear the margins (this patient's margin was anterior and beneath de-epithelialized skin so was felt not to be a candidate for re-excision). Consequently, 3/18 (17\%) of patients with invasive disease at the margin were missed during GME.

Overall, of the 51 patients who underwent gross margin evaluation, a total of 19 patients (37\%) benefitted from intraoperative gross margin evaluation. This includes the 6 out of 33 patients who underwent RRE based on GME and had a true benefit, and the 13 out of 18 patients who were correctly not recommended for RRE based on GME (Figure 1).

\section{Discussion and Conclusion}

Ongoing efforts to decrease the rate of positive margins for patients have been challenging and despite several techniques being explored worldwide, the positive margin continues to be a struggle for patients 
who choose breast conservation. Patients must then undergo additional surgical procedures which may reduce cosmesis or delay adjuvant therapy. A number of methods of reducing re-excision rates have been developed. Our institution has a longstanding practice of intraoperative gross margin evaluation with real-time re-excision based on the findings. In our experience, this process does decrease the need for a return to operating room for re-excision for those with invasive disease. A margin positive for DCIS presents an even greater challenge because it is not visualized on GME.

We performed an analysis using data collected from our Mastery of Breast Surgery CALLER registry to determine whether GME reduces re-excision rates for invasive carcinoma. Our analysis revealed an $18 \%$ reoperation rate, which compares favorably with other studies, and is below the reported national database average of $25 \%$ (6). Balch and colleagues reported a $25 \%$ re-excision rate with gross margin assessment, with tumors $<2 \mathrm{~mm}$ from a margin considered as margin positive (10). Fleming et al. (11) reports a lower re-excision rate of $9.1 \%$ with utilization of gross margin assessment, adopting a margin of 10 $\mathrm{mm}$ as an acceptable margin. Differences in definitions of margin negativity may influence re-excision rates.

Other intraoperative pathological techniques include frozen section analysis (FSA) of biopsies or cytological examination. A recent systemic review of the literature reported lower re-excision rates with FSA (12). However these methods are more time-consuming, and require further technology or training that may not be necessary if a low margin-positive rate can be achieved with gross evaluation alone.

Ultimately, the use of gross margin examination with real-time reexcision it is not as foolproof and helpful as hoped. It may result in excess tissue removal and increased operative time. Additionally, the specimen needs to be directly transferred to the pathology department for real-time consultation, which is not available in every institution. It also depends on reliable orientation of the specimen by the surgeon to ensure that the correct margin is being excised; incorrect orientation has been shown to lead to incomplete resection (13).

Therefore, each surgeon should consider this option and discuss whether it would be helpful in their institution. Because at least $37 \%$ of our patients did benefit from this process, we do plan to continue our current practice of intraoperative gross margin evaluation and remain mindful of its limitations.

Ethics Committee Approval: N/A.

Informed Consent: N/A.

Peer-review: Externally peer-reviewed.

Author Contributions: Concept - S.H., D.S.; Design - S.H., D.S.; Supervision - S.H.; Resources - S.H.; Materials - S.H., D.S.; Data Collection and/ or Processing - S.H., D.S. H.R.; Analysis and/or Interpretation - S.H., D.S., H.R.; Literature Search - S.H., D.S., H.R.; Writing Manuscript - S.H., D.S., H.R.; Critical Review - S.H., D.S., H.R.

Conflict of Interest: The authors have no conflicts of interest to declare.

Financial Disclosure: The authors declared that this study has received no financial support.

\section{References}

1. Silverstein MJ, Lagios MD, Groshen S, Waisman JR, Lewinsky BS, Martino S, Gamagami P, Colburn WJ. The influence of margin width on local control of ductal carcinoma in situ of the breast. N Engl J Med 1999; 340 : 1455-1461. (PMID: 31774979) [CrossRef]

2. Houssami N, Macaskill P, Marinovich ML, Morrow M. The association of surgical margins and local recurrence in women with early-stage invasive breast cancer treated with breast-conserving therapy: a meta-analysis. Ann Surg Oncol 2014; 21: 717-730. (PMID: 24473640) [CrossRef]

3. Vicini FA, Kestin L, Huang R, Martinez A. Does local recurrence affect the rate of distant metastases and survival in patients with early-stage breast carcinoma treated with breast-conserving therapy? Cancer 2003; 97: 910-919. (PMID: 12569590) [CrossRef]

4. Cellini C, Huston TL, Martins D, Christos P, Carson J, Kemper S, Simmons RM. Multiple re-excisions versus mastectomy in patients with persistent residual disease following breast conservation surgery. Am J Surg 2005; 189: 662-666. (PMID: 15910716) [CrossRef]

5. Heil J, Breitkreuz K, Golatta M, Czink E, Dahlkamp J, Rom J, Schuetz F, Blumenstein M, Rauch G, Sohn C. Do reexcisions impair aesthetic outcome in breast conservation surgery? Exploratory analysis of a prospective cohort study. Ann Surg Oncol 2012; 19: 541-547. (PMID: 21761099) [CrossRef]

6. Wilke LG, Czechura T, Wang C, Lapin B, Liederbach E, Winchester DP, Yao K. Repeat surgery after breast conservation for the treatment of stage 0 to II breast carcinoma: a report from the National Cancer Data Base, 2004-2010. JAMA Surg 2014; 149: 1296-1305. (PMID: 25390819) [CrossRef]

7. St John ER, Al-Khudairi R, Ashrafian H, Athanasiou T, Takats Z, Hadjiminas DJ, Darzi A, Leff DR. Diagnostic accuracy of intraoperative techniques for margin assessment in breast cancer surgery: A meta-analysis. Ann Surg 2017; 265: 300-310. (PMID: 27429028) [CrossRef]

8. Landercasper J, Attai D, Atisha D, Beitsch P, Bosserman L, Boughey J, Carter J, Edge S, Feldman S, Froman J, Greenberg C, Kaufman C, Morrow M, Pockaj B, Silverstein M, Solin L, Staley A, Vicini F, Wilke L, Yang W, Cody 3rd H. Toolbox to reduce lumpectomy reoperations and improve cosmetic outcome in breast cancer patients: the American Society of Breast Surgeons Consensus Conference. Ann Surg Oncol 2015; 22: 3174-3183. (PMID: 26215198) [CrossRef]

9. Moran MS, Schnitt SJ, Giuliano AE, Harris JR, Khan SA, Horton J, Klimberg S, Chavez-MacGregor M, Freedman G, Houssami N, Johnson PL, Morrow M. Society of Surgical Oncology-American Society for Radiation Oncology consensus guideline on margins for breast-conserving surgery with whole-breast irradiation in stages I and II invasive breast cancer. J Clin Oncol 2014; 21: 704-716. (PMID: 24515565) [CrossRef]

10. Balch GC, Mithani SK, Simpson JF, Kelley MC. Accuracy of intraoperative gross examination of surgical margin status in women undergoing partial mastectomy for breast malignancy. Am J Surg 2005; 71: 22-28. (PMID: 15757052)

11. Fleming FJ, Hill AD, Mc Dermott EW, O’Doherty A, O'Higgins NJ, Quinn CM. Intraoperative margin assessment and re-excision rate in breast conserving surgery. Eur J Surg Oncol 2004; 30: 233-237. (PMID: 15028301) [CrossRef]

12. Esbona K, Li Z, Wilke LG. Intraoperative imprint cytology and frozen section pathology for margin assessment in breast conservation surgery: a systematic review. Ann Surg Oncol 2012; 19: 3236-3245. (PMID: 22847119) [CrossRef]

13. Molina MA, Snell S, Franceschi D, Jorda M, Gomez C, Moffat FL, Powell J, Avisar E Breast specimen orientation. Ann Surg Oncol 2009; 16: 285288. (PMID: 19050966) [CrossRef] 